\title{
Criminologie
}

\section{La loi sur les jeunes contrevenants et les masse-médias}

\section{Maria Los}

Volume 20, numéro 1, 1987

Faire les nouvelles : journalisme et affaires criminelles

URI : https://id.erudit.org/iderudit/017244ar

DOI : https://doi.org/10.7202/017244ar

Aller au sommaire du numéro

Éditeur(s)

Les Presses de l'Université de Montréal

ISSN

0316-0041 (imprimé)

1492-1367 (numérique)

Découvrir la revue

\section{Citer cet article}

Los, M. (1987). La loi sur les jeunes contrevenants et les masse-médias.

Criminologie, 20(1), 7-33. https://doi.org/10.7202/017244ar

\section{Résumé de l'article}

The recent enactment of the Young Offenders Act has ended a long period of the rule of the "parens patriae", philosophy whereby the juvenile court's task was not to administer just punishment for the offence but to diagnose and treat the underlying problems. The new legislation, which follows the "justice model", attempts to broaden legal rights of accused juveniles while simultaneously making juvenile law more punitive and more focused on specific criminal offences.

The present article looks at the way the Canadian mass media reported on this major historical shift in the juvenile justice philosophy. The overt and hidden messages are analysed and the sources of the prevailing definitions explored. It has been found that the majority of informants represented powerful interest groups and organizations. Moreover, the investigated press reports created an image of fundamental consensus, and the new legislation was presented as being beneficial to the society as a whole. The inherent contradictions in the philosophy underlying the new Act were overlooked in favour of an image of a perfect balance whereby ambivalences of the new approach became transformed into virtues.

The article tests a number of hypotheses and offers theoretical interpretations of the findings. The applicability of the consensual functionalist, critical functionalist and Marxist orientations is assessed.
Ce document est protégé par la loi sur le droit d'auteur. L'utilisation des services d'Érudit (y compris la reproduction) est assujettie à sa politique d'utilisation que vous pouvez consulter en ligne.

https://apropos.erudit.org/fr/usagers/politique-dutilisation/ 


\section{LA LOI SUR LES JEUNES CONTREVENANTS ET LES MASSE-MÉDIAS* \\ Maria $\operatorname{Los}^{* * *}$}

The recent enactment of the Young Offenders Act has ended a long period of the rule of the "parens patriae", philosophy whereby the juvenile court's task was not to administer just punishment for the offence but to diagnose and treat the underlying problems. The new legislation, which follows the "justice model", attempts to broaden legal rights of accused juveniles while simultaneously making juvenile law more punitive and more focused on specific criminal offences.

The present article looks at the way the Canadian mass media reported on this major historical shift in the juvenile justice philosophy. The overt and hidden messages are analysed and the sources of the prevailing definitions explored. It has been found that the majority of informants represented powerful interest groups and organizations. Moreover, the investigated press reports created an image of fundamental consensus, and the new legislation was presented as being beneficial to the society as a whole. The inherent contradictions in the philosophy underlying the new Act were overlooked in favour of an image of a perfect balance whereby ambivalences of the new approach became transformed into virtues.

The article tests a number of hypotheses and offers theoretical interpretations of the findings. The applicability of the consensual functionalist, critical functionalist and Marxist orientations is assessed.

\section{LA LOI SUR LES JEUNES CONTREVENANTS}

Soumise au Parlement en février 1981 en tant que le Bill C-61, la Loi sur les jeunes contrevenants (LJC) fut sanctionnée le 7 juillet 1982 et promulguée le 2 avril 1984, abrogeant ainsi officiellement la Loi sur les jeunes délinquants (LJD) et faisant du Bill C-61 la version finale d'une série de propositions législatives débattues à la Chambre des communes dans les années 70 .

Cette toute récente réforme va bien au-delà de simples modifications légales ou techniques des procédures ou des définitions (à ce sujet, et

\footnotetext{
* Traduit par Geneviève Langlois.

** L'auteur est professeur au Département de criminologie de l'Université d'Ottawa. 
pour mieux saisir l'interprétation légale de la LJC, voir Bala et Lilles, 1983). En rejetant les concepts fondamentaux qui furent, à travers ce siècle, à l'origine d'une série de réformes progressives du système judiciaire pour les jeunes, la promulgation de la LJC fermait un chapitre important de l'histoire de la justice pour la jeunesse. D'approche fondamentalement non répressive, ces réformes envisageaient les jeunes délinquants plutôt comme des "enfants ayant des ennuis" que comme des «mauvais garnements", et plutôt comme des individus négligés ou mal guidés que comme des contrevenants obstinés. Leurs méfaits ne devaient pas être séparés de leurs conditions générales de vie: ainsi devaient-ils être perçus, dans leur ensemble, comme les symptômes d'un état plus généralisé de négligence, de délinquance et de dépendance. Les mesures correctives visaient davantage à améliorer cette situation complexe qu'à neutraliser les facteurs immédiats ayant pu inciter à un acte spécifique de délinquance. Les enfants n'étaient donc pas jugés entièrement responsables de leurs actes puisqu'ils ne pouvaient être blâmés des imperfections de la famille ou du milieu, deux facteurs que l'on croyait aptes à mener l'enfant à un mauvais ajustement social et à la délinquance. Par conséquent, et ayant toujours en tête le bien-être de l'enfant, la tâche du tribunal n'était pas d'administrer une peine juste pour l'infraction commise mais plutôt de déceler et de traiter des problèmes plus profonds.

La LJD de 1908, empreinte de cette philosophie du parens patriae fut perçue comme étant ce progrès révolutionnaire qui, suscitant l'humanisation du système de la justice criminelle, le rapprocha par le fait même des découvertes auxquelles des disciplines telles la psychologie du développement, la criminologie ou la sociologie en étaient arrivées. Malgré quelques critiques concernant son application dans la pratique, les principales hypothèses sur lesquelles reposait la LJD ne furent pas questionnées avant le milieu des années 60. En 1966, un comité créé par le ministère de la Justice soumettait à la Chambre des communes un rapport d'où l'on tira initialement un projet de loi, et dont la version substantiellement révisée, introduite en 1970 comme étant le Bill C-192, porta le titre de «Loi sur les jeunes contrevenants». Pour la toute première fois, l'orientation sur le traitement adoptée par la justice juvénile, le concept familier de délinquance et le paternalisme informel du tribunal pour adolescents étaient ouvertement remis en question (Fox et Spencer, 1972; Cousineau et Veevers, 1972; MacDonald, 1971; Canadian Criminology and Corrections Association, 1971).

Le projet initial de l'actuelle LJC tenta d'élargir les droits légaux des jeunes accusés tout en ramenant la législation concernée à une dimension beaucoup plus répressive et davantage axée sur certaines catégories 
d'infractions. Suivirent des projets qui adoptèrent la même politique basée sur la double responsabilité des jeunes et des agents du système de justice juvénile. De plus, dans les années 70 , la montée de la nécessité de l'implication communautaire, les idées également de diversion ainsi que la nécessité d'éviter la stigmatisation ajoutèrent à un modèle déjà fort éclectique une nouvelle dimension. L'emphase que l'on voulut mettre sur des alternatives au tribunal pour mineurs, ainsi que sur l'adoption d'éventuelles procédures officieuses de diversion entrait évidemment en conflit avec les idées admises de "due process" et de garanties de droits légaux comme avec celle qui voulait un traitement plus sévère pour les jeunes infracteurs. En d'autres termes, la flexibilité et la discrétion de ce processus de diversion avant procès sont en désaccords avec la notion établie du "modèle de justice" qui postulait des peines égales pour des infractions identiques de même que l'élimination de toute situation imprévisible et ambiguë, ou de toute discrétion.

Les écarts inquiétants entre les différents modèles et les principes qui ont marqué l'actuelle législation n'ayant jamais été résolus, la LJC présente donc un curieux mélange de propositions et de concepts contradictoires qui, malgré tout, semblent avoir été converties en qualités par le Solliciteur général l'Honorable Robert Kaplan, et ainsi reformulées en expressions traduisant "le désir d'atteindre un équilibre entre l'objectif principal de la justice criminelle qui consiste à protéger la population contre tout comportement illicite et la.volonté d'assister les jeunes gens qui entrent en conflit avec la loi " (Kaplan, 1982). Ainsi, selon ce nouveau "modèle d'équilibre", les jeunes contrevenants deviennent en même temps des adolescents dépendants et une menace pour la société, et ils sont soumis à la punition autant qu'au traitement, tout comme au due process et à la diversion arbitraire. Toutefois, toujours selon Robert Kaplan,

Cette loi concilie les intérêts de la société, le principe de la responsabilité des adolescents pour les actes qu'ils commettent, ainsi que la nature particulière des besoins et des droits de nos jeunes. Elle reflète donc les idées de notre époque et tient compte des circonstances actuelles. On ne considere plus les jeunes contrevenants comme des enfants uniquement mal dirigés ou "malades" qu'il faudrait soigner, comme c'était le cas dans le passé. Ceux-ci devraient plutôt être tenus responsables de leur conduite illicite. Cette nouvelle loi reconnaît cependant que les adolescents ne devraient pas être assimilés aux infracteurs adultes quant à leur degré de responsabilité, vu leur état de dépendance et le fait qu'ils ont moins de maturité qu'un adulte. (Kaplan, 1982) 
Il est évident que la LJD, soutenue par la doctrine du parens patriae, permit le déroulement de plusieurs pratiques injustes sous le manteau du traitement. Ceci ne rendait pas nécessairement la nouvelle Loi supérieure à la précédente. L'expertise de la philosophie et de la pratique rattachées à la LJD couplée avec l'évaluation des principes fondamentaux et des promesses de la LJC posent de nombreuses difficultés. Presque chaque aspect de la nouvelle loi peut être analysé et interprété depuis des angles variés.

Ainsi, la seule idée de modeler le tribunal pour mineurs sur son homonyme adulte peut être louable si l'on regarde la gamme des droits légaux offerts aux accusés, mais cependant remise en question par les critiques du système judiciaire pour adultes, bien conscients de ses lacunes et défauts. L'affirmation du Solliciteur général qui veut que la LJC reflète la philosophie et la situation de notre époque, même si politiquement commode, ne dévoile toutefois pas grand-chose quant à l'essence de la question. Il est à se demander, par conséquent, si des tentatives ont été faites pour informer la population canadienne de la complexité des ramifications de la nouvelle loi, complexité qui a été cachée derrière la rhétorique officielle du consensus et de l'équilibre, et derrière le jargon juridique de la loi elle-même. La présente étude se donne comme but d'examiner, à travers la presse canadienne, les comptes rendus journalistiques portant sur la LJC.

\section{PERSPECTIVES THÉORIQUES}

Dans l'analyse journalistique d'une réforme sociale les médias sont inévitablement amenés à transmettre certains messages concernant le processus inhérent à son apparition. La présentation des aboutissements sociaux suppose, dans la construction de la réalité, un processus subjectif, dans la mesure, bien entendu, où l'on développe un modèle qui, par un certain mode de structuration, puisse rendre compte de cette réalité. En conséquence tombe toujours dans le domaine du possible une multitude d'interprétations différentes, chacune pouvant éventuellement offrir une image apparemment cohérente des faits concrets de la vie. Pour les besoins de cette étude, il fut établi que l'information journalistique concernant un phénomène social comportait au moins trois importantes orientations pouvant être potentiellement identifiées comme étant 1) l'interprétation fonctionnaliste consensuelle, 2) l'interprétation fonctionnaliste critique et 3) l'interprétation marxiste critique. Quelques brèves définitions de ces trois types hypothétiques de comptes rendus journalistiques sur la LJC s'imposent. 
La perspective fonctionnaliste consensuelle soutient que l'époque était au changement et qu'il y avait un haut degré de consensus sur l'orientation de ce changement, l'ancienne loi démontrant son utilité. Les législateurs répondaient aux attentes et aux besoins sociaux, il est par conséquent normal de s'attendre à ce que la nouvelle loi réponde davantage à l'intérêt social commun et qu'elle accroisse ainsi, le degré d'intégration des adolescents dans le courant de la société. En tenant compte de cette orientation, la critique amorcée sur la LJC se dirigerait plutôt vers les critères internes dérivés soit du système judiciaire en tant que tel, soit des divers champs de la réalité officielle. Les défenseurs de l'interprétation fonctionnaliste consensuelle pourront prétendre que la LJC viole la Charte des droits, que son application est trop coûteuse, que les provinces ainsi que les bureaux de la justice pour la jeunesse ne sont pas techniquement préparés à absorber de nouvelles tâches, ainsi de suite. De surcroit, plus de temps, des moyens financiers plus considérables et une mise au point plus délicate de ce nouvel instrument juridique pourraient être demandés.

L'interprétation fonctionnaliste critique perçoit la nouvelle loi comme étant le résultat d'une prise de décision et de négociations entre certains groupes d'intérêt, leurs motifs pouvant aller des authentiques préoccupations professionnelles et des philosophies jusqu'aux intérêts purement économiques. Afin de comprendre le processus de changement, il est nécessaire d'analyser les fonctions qu'il devra remplir auprès des groupes divers ou de leurs coalitions. Il est possible, par exemple, qu'il serve à améliorer le prestige professionnel de certains groupes d'intérêt, qu'il augmente ainsi leur clientèle, qu'il leur donne plus de contrôle, plus de pouvoir, et qu'il facilite leur tâche en termes d'efforts ou de récompenses. De la même façon et dans le seul but d'exposer les raisons pour lesquelles certains groupes manifestent leur opposition et tentent de saboter la réforme établie, les journalistes peuvent tenter de montrer comment cette dernière peut devenir une menace pour la sauvegarde des intérêts de ces groupes.

Une autre interprétation offerte par les journalistes défenseurs de cette perspective consiste à faire comprendre que la promulgation d'une nouvelle loi joue un rôle essentiellement symbolique et qu'aucun changement substantiel, dans la pratique de la justice pour la jeunesse n'est vraiment attendu. Il peut être avancé que le gouvernement, afin d'assurer le support, l'intégration ou l'ordre social, de s'assurer également les votes ou encore de garantir la satisfaction de certains groupes d'intérêt, se doit de fournir la preuve d'une certaine activité, d'une certaine évolution, de créer ainsi l'impression qu'il voit constamment à l'amélioration de ses 
institutions. Les bureaucraties des tribunaux pour adolescents ainsi que celles des services à la jeunesse tendent, toutefois à fonctionner selon leur logique, leurs intérêts ou leurs idéologies professionnelles propres, et ne sont certes pas prêtes à briser leur routine. En ce sens, tout changement relèvera davantage du rituel que de la nécessité réelle.

L'interprétation marxiste postule, elle, que la nouvelle loi n'est qu'un élément parmi les efforts concertés pour renforcer la légitimité de la classe dominante, entendue cette légitimité autant dans la justice pour la jeunesse que dans le domaine politico-économique. Les journalistes à tendance marxiste mettent en évidence le fait que les racines de la délinquance des jeunes prennent naissance dans une organisation sociale injuste (pauvreté, chômage, institutions autoritaires et abusives, curriculum scolaire aliénant ainsi qu'impuissance et marginalité économique de la jeunesse). Étant donné cette réalité, l'État, en introduisant la nouvelle législation pour les jeunes, semble vouloir fixer l'attention sur les mesures légales de répression qui sont dépeintes comme les véritables solutions, plutôt que de la fixer sur les problèmes économiques et structuraux réels. La délinquance ainsi que ses méthodes d'approche sont idéologiquement façonnées en termes individuels, tout individu asocial devant être soumis à la peine appropriée afin de réformer ses comportements. Rien d'autre n'est à changer. À l'argument selon lequel tous les individus - incluant les adolescents — bénéficient des mêmes droits pour se défendre contre une accusation mal fondée ou un jugement trop sévère, on pourrait répondre qu'il s'agit là d'une tentative de la part de la classe dirigeante, visant à promouvoir le mythe d'une société sans classes, où tous les individus sont égaux, leurs actes criminels n'étant que le résultat d'échecs individuels.

Toute hypothèse qui voudrait traiter de l'orientation dominante des journalistes canadiens doit d'abord être fondée sur une certaine perception du rôle et de la nature des médias canadiens. Parallèlement aux perspectives précédemment étudiées en tant que modes d'interprétation de la réalité sociale, il est une fois de plus possible de distinguer trois interprétations.

L'interprétation fonctionnaliste consensuelle suggère le fait que les médias contribuent à l'autovigilance d'une société: son potentiel, ses problèmes, ses forces, ses éventuelles stratégies de changement, etc. Les médias présentent donc les vues et intérêts des divers groupes sociaux ainsi que ceux et celles des organisations. De plus, ils intensifient l'entente mutuelle entre ces groupes et voient à l'achèvement d'une certaine forme d'équilibre des intérêts. Ainsi, les membres de la société prennent-ils conscience, à travers l'information qui offre la représenta- 
tion objective des valeurs et des intérêts dominants, de leur sort commun et de leur interdépendance. Les médias s'occupent, en somme, de promouvoir une vraie communication et intégration sociales, de la même façon qu'ils assistent la population dans son ajustement aux nombreuses réalités et complexités de la société moderne.

L'interprétation fonctionnaliste critique postule, à son tour, que les médias tendent à représenter les vues, les préférences et les intérêts de ces groupes qui bénéficient d'une plus grande influence politique (par exemple, le gouvernement, les groupes de pression puissants, etc.) et qui, en même temps, sont plus vigoureux et plus efficaces lorsqu'il s'agit de projeter leurs opinions. Puisque leurs positions sociales ou politiques font deux les cibles les plus attrayantes pour les médias, les journalistes seront davantage enclins à présenter les visions et les définitions que ces groupes donneront aux situations nouvelles ou problématiques. Lorsque, souvent, les journalistes eux-mêmes s imbriquent à l'intérieur de ces regroupements élitaires, il est aisé de comprendre que l'entretien de certains liens personnels ou encore que la simple compréhension mutuelle rende le choix des individus à interviewer plus facile à faire. Par conséquent, à cause de la structure pluraliste et inégalitaire de la société moderne qui entraîne nécessairement une distribution inégale de l'influence politique, la fonction des médias comme représentants de la socićté peut être amenée à être déformée.

Afỉn de veiller à leur survivance même, les entreprises d'information se doivent dentretenir des relations amicales avec le monde des affaires, un autre facteur dont l'impact sur la nature et le fond des reportages journalistiques est tout à fait évident. Car non seulement les propriétaires de ces entreprises sont-ils eux-mêmes des membres du secteur des affaires, mais plus encore la publicité commerciale constituet-elle, pour ces dernières, la source de revenu la plus importante. Il est dès lors évident que les masse-médias, évitent tout ce qui pourrait offenser ou leur mettre à dos ce secteur commercial, sans toutefois s'engager à promouvoir activement leurs intérêts. Puisque repose sur la popularité d'un journal le succès même du publicitaire, ses annonces ont tout intérêt à être vues par le plus grand nombre de lecteurs possible, ses soucis professionnels tendront à le ramener vers ces intérêts économiques également partagés avec certains propriétaires de journaux et, indirectement avec ceux de leurs employés. En retour une tension puissante s"exerce sur les journalistes dans la mesure où ils doivent sans cesse chercher les stratégies qui maximiseront la vente de leur média. Pour atteindre cet objectif ils tentent de s'approprier le plus grand nombre d'acheteurs potentiels (par exemple, la classe moyenne conservatrice, les adoles- 
cents, les ménagères), et ils ajustent la nature de l'information à transmettre selon la demande et les préférences marquées de ces groupes.

La théorie fonctionnaliste critique assume l'idée que le comportement des individus, des groupes et des organisations de la société industrielle moderne sont, dans une large mesure, formés par les pressions et les opportunités économiques. Ils recherchent des opportunités à maximiser leurs revenus et, s'ils font face à des résistances, on doit s'attendre à une réponse déviante. En ce sens, il est compréhensible que les médias ajustent leur information selon les désirs de l'éventuel abonné, dans la mesure où ils doivent s'approprier la plus grande part du marché des lecteurs.

Finalement, l' interprétation marxiste supporte l'idée que les massemédias sont partie intégrante de la superstructure de la société de classes et que leur mission principale est de maintenirl' hégémonie et la légitimité de l'ordre établi alors que, à long terme, ils visent à assurer la domination continue de la classe capitaliste. Afin de soutenir leur crédibilité, les médias doivent montrer des apparences d'objectivité et d'indépendance; ils doivent même, dans certains cas, critiquer et mettre à jour certaines pratiques injustes du pouvoir en place. Malgré tout, leur tâche est de contribuer, à long terme, à la vulgarisation de l'idéologie dominante. Faisant partie d'un réseau complexe d'institutions éducationnelles, culturelles et légales qui se sont développées sur les bases de la structure économique existante, les principaux médias ne peuvent oublier leur loyauté envers l'ordre dominant sans perdre leur viabilité économique et leur place structurellement déterminée dans la société.

\section{HYPOTHĖSES DE RECHERCHE}

La présente étude postule que l'interprétation fonctionnaliste consensuelle du rôle et de la nature des masse-médias est considérablement éloignée de la réalité canadienne (hypothèse 1). Dans cette étude, les indicateurs qui permettent d'évaluer l'exactitude de l'interprétation ci-haut mentionnée seront: une représentation équitable, dans les rapports journalistiques sur la LJC, des adolescents et des délinquants eux-mêmes; une représentation équilibrée des différents groupes impliqués dans les instances administratives de la justice pour adolescents (juges, avocats, travailleurs sociaux, etc.) ainsi que des chercheurs ou des avocats des droits de la personne. De plus, on devrait, préalablement à la promulgation de la LJC, fournir à la population les différentes opinions sur les buts, la philosophie et les procédures proposées dans le projet de loi C-61. L'évaluation de ce genre de reportage journalistique, 
par divers groupes, allant des adolescents jusqu'aux politiciens, serait présentée. On devrait faire une tentative réelle pour aller au-delà de la rhétorique officielle et pour stimuler les discussions entourant le véritable contenu et le véritable sens du Bill. Des membres de la population se verraient également donner l'opportunite d'exprimer leur point de vue, de clarifier la "fonctionnalité" de la nouvelle loi pour la société dans son ensemble, et, éventuellement, d'atteindre un état consensuel bien renseigné.

Il est postulé, également, dans un deuxième temps, que l'interprétation fonctionnaliste critique s'applique relativement bien à la réalité du reportage canadien (hypothèse 2 ). Les indicateurs pertinents devraient comprendre: l'utilisation systématique d'informateurs qui appartiennent à certains groupes d'intérêt dont l'influence est certaine (juges, policiers, avocats) ou qui représentent le gouvernement tout comme on devrait négliger systématiquement les opinions de groupes non organisés et impuissants comme les adolescents, les délinquants eux-mêmes ainsi que leurs parents. Du reste, puisque la question de la LJC n'est pas d'une importance majeure pour la population, l'information sur la question devrait être relativement rare, courte et ne devrait pas se retrouver parmi les grands titres des premières pages. Quelques reportages de type sensationnel concernant la délinquance ou la montée des infractions violentes devraient accompagner les reportages sur la loi, et plus tard sur sa promulgation. La création d'un sentiment d'intimidation accompagnée de la promesse d'une loi plus dure peuvent être vues comme autant de tactiques efficaces pour attirer l'attention des lecteurs.

On pourrait considérer qu'employer les services d'informateurs influents supporte l'interprétation marxiste. Peut-être pourrait-on avancer que cette orientation laisse entendre que les médias doivent faire plus d'efforts afin d'assumer l'image de reportages objectifs qui incluraient les opinions des chercheurs plus critiques, celles des défenseurs des droits de l'homme, celles des représentants du NPD, etc. Le message général, toutefois, devrait ètre en accord avec la législation qui fournit une définition purement légale d'un problème abritant des dimensions sociales et économiques beaucoup plus complexes.

En gardant bien en tête que, dans le contexte de cette recherche, les indicateurs empiriques qui supportent les interprétations marxiste et fonctionnaliste critiques apparaissent assez similaires, la présente étude ne se donne pas comme but de produire les preuves qui permettraient de confirmer l'une plutôt que l'autre. Si, comme les fonctionnalistes critiques l'affirment, qu'il est vrai que les médias voient avant tout à maximiser leurs revenus, il peut être postulé que l'on s'attendra que 
l'emphase soit mise sur les mesures de punition inhérentes à la nouvelle loi. Plusieurs études portant sur l'opinion publique indiquent que les Canadiens supportent aisément l'idée de mesures plus strictes de punition et qu'ils sont contrariés par le concept d'indulgence envers les contrevenants. En retour, l'interprétation selon laquelle les médias servent principalement à promouvoir l'idéologie dominante justifierait cette prédiction qui voudrait les voir insister sur la disposition, pour tous les individus, de droits égaux, en même temps que sur l'importance de la responsabilité individuelle, puisque ce sont là les composantes principales de l'idéologie dominante d'une société capitaliste libérale et démocratique. D'un autre côté, puisque le modèle de just desert et l'emphase mise sur la "punition équitable" vont dans le sens des valeurs relatives à l'individu dans cette idéologie, il devient extrêmement délicat d'essayer de prouver empiriquement la supériorité d'une de ces théories sur l'autre. En conclure quelque chose ne serait possible que dans la mesure où l'on pourrait avancer d'autres arguments a priori relatifs à la nature "globalisante" de l'organisation sociale et de la place structurellement occupée par les médias dans cette organisation. Que les masse-médias renforcent la structure de l'autorité établie en même temps qu'ils appuient le statu quo ne permet que d'avancer l'idée selon laquelle il s'agit bien là d'une preuve partielle visant à corroborer chacune des deux théories. De telles découvertes sont incapables de jeter la lumière sur l'analyse du statu quo qui profite des messages livrés par les médias.

Il semble que l'orientation fonctionnaliste critique, telle que ci-haut présentée, est plus opérationnalisable et plus facilement vérifiable empiriquement que l'analyse marxiste. L'hypothèse 2 se réfêre à cette version empirique relativement simple de l'approche fonctionnaliste critique, et ne porte aucun jugement sur la nature exacte des relations sociales dominantes. D'un autre côté, et afin de guider nos explications de la réalité sociale, la théorie marxiste de la structure sociale doit être acceptée $a$ priori même si elle ne peut pas être réduite à des propositions empiriquement vérifiables et même s'il se révèle futile d'essayer de la mettre à l'épreuve dans le contexte de cette recherche. En somme, il est à prévoir que l'analyse des reportages de presse sur la LJC confirmera l'influence que certains groupes d'intérêt peuvent avoir sur la représentation de cette réforme, confirmera également que les médias renforcent de façon générale la structure de crédibilité établie, mais l'analyse ne sera pas capable de prouver ni de réfuter la subordination des médias ou des groupes de pression concernés aux intérêts dominants de la classe dirigeante (hypothèse 3). 
Les trois dernières hypothèses traitent du contenu et de l'orientation actuelle des comptes rendus journalistiques sur la LJC. Une d'entre elles postule que le modèle fonctionnaliste-critique du rôle des médias dans la société est une représentation relativement exacte de leur rôle dans les reportages sur la LJC. Si c'est le cas nous devons nous attendre que les reporters, dont les articles sont basés sur des entrevues avec des membres de groupes influents, ne mettront pas en évidence leurs intérêts particuliers, mais vont plutôt décrire leurs préoccupations comme universelles et orientées vers leurs conceptions du bien commun. Nous devons donc nous attendre à ce que l'interprétation, par les médias, des événements entourant la LJC ressemble fort à la vision offerte par le fonctionnalisme consensuel (hypothèse 4).

Selon cette perspective, tout conflit qui peut survenir est vu comme relié à un désagrément de second ordre, comme par exemple le manque de ressources ou des problèmes techniques relatifs à l'implantation de la loi. Il est prédit que presque aucune évidence ne sera trouvée permettant de suggérer que l'approche fonctionnaliste critique est une démarche commune parmi les journalistes étudiés ici (hypothèse 5). Par exemple, aucun effort ne fut fait pour identifier les intérêts particuliers des groupes influents, il ne fut fait aucunement mention de la possible fonction symbolique ou ritualiste de la nouvelle loi ou encore de la possibilité de sa neutralisation bureaucratique, et il y eut peu de déclarations sur les relations fonctionnelles entre l'inégalité sociale et la délinquance.

Finalement, il est suggéré que l'analyse de type marxiste est absente des rapports journalistiques sur la LJC. En d'autres termes, aucun argument concernant l'élaboration idéologique du problème ainsi que sa solution ne peut être trouvé dans le matériel étudié (hypothèse 6).

\section{LA RECHERCE SUR LES MÉDIAS ÉCRITS}

La présente recherche implique une analyse détaillée des articles qui ont été écrits, entre 1980 et 1984, sur la LJD, la LJC et sur la délinquance des jeunes en général. Ces articles ont été sélectionnés dans deux quotidiens anglais, deux quotidiens français, un magazine hebdomadaire anglais et un magazine hebdomadaire français. La période quinquennale couvre les deux années et demie qui précédèrent la promulgation de la LJC, presque les deux années de préparation pour sa mise en exécution et les neuf mois de son actuelle application. Voici une brève description de l'échantillon des médias. 
En anglais:

The Globe and Mail (GM) est un quotidien torontois à tirage national (circulation: 339200 copies) appartenant à Thomson Newspaper Ltd. Cet empire de l'information est propriétaire de 40 quotidiens canadiens, de 12 hebdomadaires, de même que de 71 quotidiens et de 5 hebdomadaires américains (voir Siegel, 1983: 112; Royal Commission..., 1981: 242-244).

Le Toronto Star (TS) également un quotidien torontois, avec ceci de particulier qu'il a le plus grand tirage de tous les joumaux de langue anglaise (circ 514300 copies). Il est la propriété de Torstar, impliqué dans la publication de 27 joumaux communautaires, de nombreux hebdomadaires, de livres et d'autres entreprises d'information (voir Siegel, 1983: 112, 119; Royal Commission..., 1981: 98-99).

Maclean's (ML) est un magazine d'information hebdomadaire (circ. 639500 copies) (Audley, 1983: 71).

\section{En français:}

Le Devoir (LD) est un quotidien indépendant, il est publié à Montréal et il s'adresse à une élite intellectuelle (circ. 42500 copies) (voir Siegel, 1983: 107, 115; Royal Commission..., 1981: 29, 100).

La Presse (LP) est un quotidien publié à Montréal (circ. 208300 copies) qui appartient à Trans-Canada Newspapers, lequel publie 4 quotidiens et contrôle 32\% du marché de langue française (Siegel, 1983: $114,116)$.

L'actualité (LA) est un magazine d'information hebdomadaire (circ. 235800 copies) (Audley, 1983: 71).

Le nombre des articles consultés pour la période de 5 ans: The Globe and Mail - 54; le Toronto Star - 45; Maclean's - 5; Le Devoir - 40; La Presse - 20; L'actualité - 0 (total de 164 articles). 
5. RÉSULTATS DE LA RECHERCHE: ÉVALUATION DE LA LJD ET DE LA LJC ${ }^{1}$

La LJD fut mentionnée dans 53 articles, représentant ainsi $32,3 \%$ de tous les articles étudiés $(43,3 \%$ étaient en anglais et $15,4 \%$ en français). Alors que l'on ne pouvait trouver que six arguments en faveur de la LJD, ses caractéristiques négatives furent mentionnées 98 fois. Voici comment étaient distribués les arguments contre la LJD: $42,9 \%$ des 98 arguments pointèrent le fait que les concepts mêmes de délinquance (incluant les infractions statutaires, etc.) et de tribunal pour adolescents (paternaliste, derrière les portes closes, etc.) étaient vieillis. De plus, 32,6\% des arguments firent référence aux trop peu nombreuses protections légales, aux sentences indéterminées, aux conflits avec la Charte des droits et à la trop grande liberté laissée à la police ainsi qu'aux juges, etc. Finalement, $15,3 \%$ de tous les arguments soulignèrent que la LJDétait trop laxiste, qu'elle n'insistait pas assez sur la responsabilité et qu'elle reposait sur l'approche du traitement, chose qui n'était pas appropriée pour les jeunes contrevenants endurcis.

Les articles qui se chargèrent d'analyser la LJD mentionnèrent les points de vue de 95 personnes ou organisations. Le tableau suivant présente les pourcentages des différentes personnes consultées à propos de la LJD:

\begin{tabular}{lc}
\hline & $\%$ \\
Juges & 35,8 \\
Fonctionnaires (fédéraux/provinciaux) & 25,2 \\
Avocats & 18,0 \\
Chercheurs & 9,4 \\
Policiers & 4,2 \\
Travailleurs sociaux & 3,2 \\
M.P. & 3,2 \\
Commissaires d'écoles & 1,0 \\
Total & $100,0 \%(\mathrm{~N}=95)$ \\
\hline
\end{tabular}

Aucun adolescent, aucun parent, aucun professeur ou travailleur communautaire ne furent interviewés. C'est dans le G.M., où $30,4 \%$ de

1. Alors que cet article présente l'analyse générale des résultats les plus importants, la question des différences entre les reportages journalistiques observées au Québec et en Ontario concernant la LJC, en même temps que la représentation, par les médias, de la Loi, seront explorées dans des publications ultérieures, toujours menées dans le cadre de cette recherche. 
toutes les opinions émises étaient gouvernementales, que l'on rencontra le plus souvent la confiance mise dans les avis du gouvernement. Les pourcentages respectifs pour L.D. et le T.S., concernant la même question, étaient de $25 \%$ et de $10 \%$. L.P. n'interviewa qu'une seule personne: un policier.

De tous les articles portant sur la LJD, 29 affichaient des titres représentant clairement les opinions d'individus particuliers ou de groupes. Presque $49 \%$ de ces titres faisaient référence aux points de vue des juges et $21 \%$ aux déclarations gouvernementales (incluant $13,8 \%$ de références à l'opinion du Solliciteur général lui-même).

La LJC fut mentionnée, elle, dans 69 articles (constituant $42,1 \%$ de tous les articles; constituant également $52,9 \%$ des articles en langue anglaise et $23,3 \%$ de ceux en langue française). En tout, 166 arguments positifs furent présentés aux lecteurs; $37,4 \%$ de ceux-ci faisait l'éloge de la LJC parce qu'elle fournissait des droits légaux aux jeunes contrevenants (procès en bonne et due forme, droit à un avocat, caution, appel, détention séparée des adolescents, etc.), parce qu'elle avait aboli les sentences indéterminées ainsi que les infractions statutaires et, finalement, parce qu'elle permettait au public d'examiner rigoureusement les procédures du tribunal. De plus, $22,9 \%$ des arguments positifs faisaient allusion de façon plus ou moins précise au fait que la LJC était meilleure et généralement plus en "accord avec l'époque" que la LJD. La modification quant aux limites d'âge (de 7 à 16 ans devenues de 12 à 18 ans) fut approuvée par $20,5 \%$ des opinions émises. L'insistance sur la part de responsabilité qui incombe à l'adolescent fut louangée dans $10,2 \%$ des cas et le souci de la LJC concernant la protection de la société, fut mentionnée dans $3,6 \%$ de tous les cas d'évaluation positive. Les opinions autres que celles soulignées ici ne représentèrent que $5,4 \%$ du total.

Les évaluations négatives de la LJC ont été exprimées dans 57 déclarations clairement défavorables mais, il doit être noté que la majorité de ceux ayant ainsi fait leurs commentaires affirmèrent néanmoins soutenir de façon générale la législation concernée ainsi que ses principes fondamentaux. Isolée, la plus grande catégorie d'opinions négatives $(25,8 \%)$ se disait concernée par la proposition, fort controversée, d'élever l'âge maximum des contrevenants sous la juridiction du tribunal pour adolescents (incluant la proposition antérieure qui voulait laisser aux provinces le soin de trancher cette question, laquelle fut jugée par maints critiques comme entrant en conflit avec la Charte des droits). Un autre 
souci fréquemment signalé relevait du fait que l'implantation de la LJC s'avérait fort coûteuse, surtout en ayant élevé l'âge maximum des jeunes contrevenants $(24,6 \%)$. S'alarmaient également quelques critiques du fait de l'élimination d'une pratique qui relevait, parmi les procédures du tribunal pour adolescents de la tradition: le huis clos $(17,7 \%)$, leur souci principal étant que la publicité pouvait éventuellement nuire aux jeunes suspects. Les plaintes au sujet de la trop grande générosité quant à l'éventail des droits offerts aux jeunes délinquants, l'indulgence générale de la LJC et l'augmentation de l'âge minimum de la responsabilité de 7 à 12 ans, comptèrent pour $16,2 \%$ de toutes les opinions défavorables. L'insuffisante garantie des droits légaux accordés aux jeunes contrevenants (par exemple, dans le cas de l'éligibilité à l'aide juridique ou dans celui de l'application arbitraire de certaines mesures alternatives avant procès) fut soulevée dans $6,4 \%$ de tous les commentaires critiques. Ce qui resta comme opinions défavorables $(8,1 \%)$ fit référence à d'autres aspects de la nouvelle législation.

Les coûts de l'application de la LJC se virent allouer plus d'une attention après sa sanction, en 1982, 33 articles (91\% desquels avaient été publiés dans la presse anglophone) discutaient ce problème en détail ou le mentionnait, particulièrement en ce qui concerne le partage des dépenses entre le gouvernement fédéral et les gouvernements provinciaux.

Les représentants des gouvernements provinciaux et du gouvernement fédéral comptèrent pour $43,2 \%$ des organisations et des individus qui exprimèrent leur opinion sur la LJC (incluant des citations du Solliciteur général, les opinions émises par Robert Kaplan: $20,6 \%$; des références faites à des représentants des gouvemements provinciaux: $18,5 \%$ ainsi que des références faites à des fonctionnaires fédéraux autres que Kaplan: $4,1 \%$ ). Les membres des professions juridiques comptèrent pour $18,5 \%$ des opinions citées (opinions des juges: $11 \%$; celles des avocats de la défense: $7,5 \%$ ). Les travailleurs sociaux $(8,9 \%)$ et les fonctionnaires de l'éducation $(6,8 \%)$ justifièrent un autre $15,7 \%$. Les membres du Parlement furent mentionnés dans $9.6 \%$ de tous les renvois à la LJC (NPD: $5,5 \%$ et PC: $4,1 \%$ ). Finalement, les représentants de la police furent consultés dans $6,2 \%$, et les chercheurs dans $5,5 \%$ de tous les cas où des opinions étaient résumées.

Quarante-huit articles sur la LJC affichaient des titres qui présentaient l'opinion d'une personne spécifique et la majorité faisait référence à des fonctionnaires gouvernementaux $(62,5 \%)$, parmi lesquels c'est Robert Kaplan $(37,5 \%)$ qui attira le plus d'attention. Les opinions des représentants de la profession légale se trouvèrent mentionnées dans 
$22,9 \%$ des titres (juges: $14,6 \%$, avocats: $8,3 \%$ ). Les opinions des chercheurs n'apparaissaient que dans 2,1 des titres qui furent identifiés comme la représentation manifeste de déclarations faites par des individus spécifiques.

Enfin, quelques mots clés ont été identifiés et leur fréquence d'apparition dans chacun des articles traitant de la LJC examinée. Il en est ressorti que, premièrement, le nom du Solliciteur général, Robert Kaplan, apparut 121 fois dans les articles qui, soit débattaient la question de la LJC, soit n'en faisaient qu'une brève mention (la fréquence moyenne de l'apparition de son nom étant de 1,8 fois par article). Les droits des jeunes contrevenants (des mots tels "autorisations légales", "privilèges", "garanties») furent cités 75 fois, soit une fréquence de 1,1 fois par article, et le concept de responsabilité des jeunes contrevenants, 38 fois $(0,6$ par article) dans un ensemble de 69 reportages. Seulement 13 déclarations $(0,2$ par article) ont stipulé que les jeunes contrevenants auraient à faire face, sous la nouvelle législation, à des conséquences plus sérieuses tandis que l'on observa 5 fois seulement l'idée selon laquelle le "modèle de justice» était le principe conducteur de la LJC. Enfin, des expressions indiquant un haut degré de consensus (telles, "presque tous en conviennent", «en accord avec l'époque", "dans l'intéret de la société") furent rencontrées 22 fois dans les 69 articles traitant de la LJC

\section{RÉSULTATS DE RECHERCHE: PORTRAIT DE LA DÉLIN- QUANCE}

Les journaux étudiés ne portèrent guère d'attention à la délinquance des jeunes, à part ces quelques-uns dont les articles faisaient ordinairement mention des taux, ascendants ou descendants, de la délinquance. Ressortant de l'échantillon des 164 articles dépouillés, 39 d'entre eux affirmaient que la délinquance des jeunes augmente et 6 qu'elle avait tendance à diminuer. Parmi les articles discutant de l'augmentation, on retrouvait une proportion équilibrée entre ceux écrits en anglais et ceux écrits en français. Par contre, les articles mentionnant une diminution (6 articles) étaient tous publiés dans des journaux anglophones. Les journaux les plus populaires (LP et le TS) ainsi que l'hebdomadaire anglophone ML ont présenté un taux beaucoup plus élevé de reportages suggérant une hausse des infractions commises par les adolescents (respectivement $35 \%, 42 \%$ et $80 \%$ ) que ceux aux ambitions plus élevées qui s'adressent à des lecteurs plus instruits, nous parlons de LD et du GM (20\% et $4 \%$ de tous les articles sur la délinquance des jeunes publiés dans ces journaux entre 1980 et 1984). La très grande majorité des reportages 
joumalistiques faisait référence à des situations locales, dans une ville ou dans une province, et n'établissait aucune généralisation sur la tendance de la délinquance dans l'ensemble du pays ou en Amérique du Nord en général.

Des 77 types d'infractions que l'on croyait à la hausse, $37,7 \%$ étaient des crimes violents ou sérieux, $26 \%$ des activités délinquantes d'ordre général et non spécifiées, $13 \%$ des infractions à la propriété et $9,1 \%$ des violations reliées à la drogue. Parmi les 80 causes de la hausse de la délinquance mentionnées dans les articles, $50 \%$ impliquaient de graves problèmes sociaux (pauvreté: $12,5 \%$; chômage: $11,3 \%$; services sociaux insuffisamment financés et pénurie de moyens dans l'aide à l'enfance: $10 \%$; manque de débouchés offerts par une carrière: $7,5 \%$ et un haut coût des loisirs: $3,8 \%$ ). De plus, $27,5 \%$ identifièrent les causes de la délinquance comme reliées à la structure familiale (rupture familiale, divorce et famille monoparentale: $12,5 \%$; enfants maltraités et indifférence des parents: $10 \%$; travail extérieur de la mère: $5 \%$ ). Finalement, $15 \%$ de tous les facteurs que l'on croyait aptes à contribuer à la hausse de la délinquance étaient reliés au laxisme social en général $(7,5 \%)$ et à l'indulgence du système judiciaire pour adolescents en particulier $(7,5 \%)$.

Concernant les jeunes délinquants, il fut possible de distinguer 5 stéréotypes qui sont apparus fréquemment dans les reportages étudiés. Sur 36 références à ces stéréotypes, $33,3 \%$ suggèrent que les délinquants sont de plus en plus jeunes, $22,2 \%$ que les infractions commises par ces jeunes devenaient de plus en plus sophistiquées, $19,4 \%$ déclarèrent que les délinquants, sachant manipuler le système judiciaire, s'en moquaient éperdument, $16,7 \%$ insistèrent sur le fait que les jeunes contrevenants étaient exploités par les criminels adultes (surtout des receleurs) et enfin, $8,4 \%$ montrèrent que certains cercles hautement organisés de jeunes étaient typiques de la délinquance. Le TS avec ses histoires sensationnelles relatives à la délinquance observée dans la région métropolitaine de Toronto, a été le plus fréquent à offrir ses stéréotypes aux lecteurs. La plupart de ces histoires furent publiées en 1980-1981, cette période précédant la sanction de la LJC.

\section{D'AUTRES RÉSULTATS DE RECHERCHE: SOURCES ET PRÉSENTATION DES OPINIONS}

Il fut établi que les titres d'environ $90 \%$ des articles répertoriés n'induisaient pas le lecteur en erreur et qu'ils traduisaient assez bien la matière traitée: c'est le TS qui se retrouva avec la proportion la plus 
élevée ( $80 \%$ ) des articles n'observant pas ce critère ayant, avec le ML, le plus de titres à caractère sensationnel: respectivement $51 \%$ et $60 \%$. Les proportions, pour les journaux qui restèrent n'était que de $6 \%$. Dans la mesure où l'on essaya de présenter différents points de vue et de pointer les aspects controversés de la question, les articles offerts par le ML et LD avaient tendance à être davantage orientés que ceux publiés dans les autres journaux. Les articles classés dans cette catégorie constituèrent $60 \%$ de tous ceux du ML, dans la même veine, $L D$ en avait $55 \%$, le GM $46 \%$, le TS $40 \%$ et le LP seulement $15 \%$. Lorsque, toutefois, le message était pris dans son ensemble, $80 \%$ des articles du ML reflétaient un haut degré de consensus au niveau de la discussion; le TS ramassant $53 \%$ de tels articles; le GM, 50\%; LP, $35 \%$; et LD, $25 \%$. Il est bon de souligner que les «reportages à opinions multiples» réfêrent, à propos d'un débat donné, à plus qu'une seule vision des choses. Il fut dès lors établi que les points de divergence tendaient plutôt à être reliés aux aspects marginaux d'une question, et à être formulés, de la part de ceux qui rendaient une opinion opposée, par des déclarations assez simples. Un large éventail d'autres opinions ne furent pratiquemment pas retenues.

On essaya également d'indiquer dans quelles proportions les hommes et les femmes furent consultés pour émettre leurs opinions sur la question de la délinquance des jeunes et sur celle de son contrôle juridique. En ce sens, l'on démontra que dans les quotidiens environ $10 \%$ des informateurs étaient des femmes, la proportion respective pour le ML étant de $21 \%$. Lorsqu'on considéra les photographies accompagnant les articles, l'on constata que les femmes constituaient $45 \%$ de toutes les personnes photographiées dans le LP, $25 \%$ dans LD, $11 \%$ dans le TS et $6 \%$ dans le GM. Ce modèle de distribution dégage le fait que si les journaux francophones négligent les opinions de femmes autant que les journaux anglophones, il en ressort néanmoins que les premiers ont davantage tendance à considérer ces dernières comme des images décoratives.

De la même façon, les adolescents, dont les opinions étaient presque totalement ignorées par les journalistes se voyaient néanmoins allouer, par les journaux francophones, une place importante en ce qui a trait à la représentation photographique: dans LP, $36 \%$ de toutes les personnes ainsi représentées étaient des adolescents, $33 \%$ dans LD, $20 \%$ dans le TS, $6,3 \%$ dans le GM, 44,6\% dans le ML. En tout, 67 individus furent représentés dans les photos accompagnant quelques-uns des articles. Voici comment se répartissait les diverses catégories auxquelles ces personnes appartenaient: 


\begin{tabular}{lc}
\hline & $\%$ \\
Adolescents & 29,9 \\
Fonctionnaires gouvernementaux & $19,4(\mathrm{R}$. Kaplan 7,5$)$ \\
Policiers & 13,4 \\
Juges & 9,0 \\
Chercheurs & 8,9 \\
Spécialistes de l'enfance/ & \\
travailleurs sociaux & 7,5 \\
MP & 7,5 \\
Total & $100,0 \%(\mathrm{~N}=67)$ \\
\hline
\end{tabular}

Finalement, une analyse globale des types préférés d'informateurs interviewés ou simplement cités dans les articles concernés par la délinquance des jeunes et par ses lois appropriées fut entreprise. Des 431 individus étudiés, $25,5 \%$ étaient des représentants de professions juridiques (juges: $15,8 \%$; avocats de la défense: $5,8 \%$; avocats de la couronne: $3,9 \%$ ), 24,1\% des fonctionnaires gouvernementaux (au provincial: 15,5\%; Robert Kaplan: 7\%; et autres représentants du gouvernement fédéral: $1,6 \%$ ). Les travailleurs sociaux ou de l'éducation, les spécialistes de l'enfance et les conseillers municipaux comptèrent pour $16,1 \%$ du total. La police constitua $11,8 \%$ des sources pour les journalistes; les MP, $11,4 \%$; les chercheurs, $7,2 \%$; et les autres, $3,9 \%$. Alors que LD sollicitait les opinions des chercheurs dans $15,7 \%$ de tous les cas où des informateurs de l'extérieur étaient consultés, les proportions respectives pour les autres journaux étaient négligeables. Lorsque ces données sont regroupées dans des catégories encore plus générales, il apparaît que les représentants du système judiciaire criminel comptaient pour $37,3 \%$ de tous les individus dont les opinions furent présentées aux lecteurs, alors qu'un $35,5 \%$ était alloué aux membres du gouvernement et du Parlement.

La distribution des informateurs qui représentaient divers partis politiques méritait également un examen plus minutieux, et c'est ainsi qu'en tout, les opinions des membres du Parlement et des assemblées provinciales furent citées 49 fois ( 36 fois par des journaux francophones et 13 fois par leurs homonymes anglophones). Parmi ces 49 individus, 15 étaient des membres du Parlement ( 9 d'entre eux représentant le NPD, 5 le PPC et un le PL) et 34 des membres de la législature provinciale du Québec (24 du PQ et 10 du PLQ). 


\section{DISCUSSION: MESSAGES DÉCLARÉS ET MESSAGES CACHÉS}

Les reportages journalistiques sur la LJC et sur la question de la délinquance des jeunes sont peu abondants, routiniers, superficiels, empreint de dilettantisme et ils relèvent souvent de la répétition. Aucune tentative ne fut amorcée pour essayer d'expliquer la philosophie et les différents buts conflictuels au sein de la L.J.C.. On n'indiqua pas également que la philosophie du traitement et de l'aide était rejetée en faveur du modèle de just desert. L'idée de chercher des moyens plus efficaces en vue de mettre l'ancienne philosophie en pratique ne fut pas envisagée; dès lors, il est possible que la vague présentation de la nouvelle loi, basée sur des clichés dénués de sens et relatant la rhétorique officielle, ait, en fait, été désignée pour satisfaire les diverses attentes des lecteurs. Ainsi, alors que ceux qui s'inquiètaient des libertés civiques étaient réassurés par l'expansion considérable de la nouvelle loi quant aux droits des jeunes contrevenants, ceux en faveur d'une répression et d'une discipline plus strictes étaient également satisfaits par la place importante que l'on sut donner aux slogans du genre «sécurité du public» ou «responsabilité des jeunes contrevenants».

Alors que les deux magazines d'informations hebdomadaires accusaient un manque significatif d'intérêt pour la LJC, les quotidiens anglophones dirigèrent, par rapport aux quotidiens francophones, davantage leur attention sur la nouvelle législation. LD et LP semblèrent plus préoccupés par la Loi de la protection de la jeunesse québécoise que par la législation fédérale. Malgré les critiques sévères adressées à leur loi provinciale, ils ne tentèrent pas de chercher conseil auprès du gouvernement fédéral et, à la place, ils projetèrent une image puissante de l'autonomie juridique de leur province.

La proportion des opinions négatives par rapport aux positives portées sur la LJC fut de 1 à 2,7 en faveur des avis positifs. Un grand pourcentage des arguments négatifs publiés durant la période qui précéda la promulgation de la Loi était concentré sur la proposition qui avait été faite de laisser à la discrétion des provinces le choix de l'âge maximum. Lorsque cette critique fut accommodée dans la version finale de la loi, tout en manifestant encore de nombreuses oppositions concernant les nouvelles limites d'âge, les sentiments négatifs se concentrèrent par-dessus tout sur les problèmes financiers entraînés par l'application de la LJC. Le souci de trouver la meilleure formule possible pour assurer le partage des coûts entre le gouvernement fédéral et les gouvemements provinciaux étouffa toute crainte additionnelle au sujet de la loi elle-même. 
Nous devons nous rappeler, toutefois, que n'importe quelle forme de communication, les rapports de presse ne faisant pas exception à la règle, possède différents sens qui, au premier abord, ne sont pas nécessairement évidents. Lorsque l'on analyse un texte écrit, il est important de voir au-delà des déclarations "ouvertes» et interpréter le message implicite adressé aux lecteurs: par exemple, tant les messages omis que suggérés doivent être étudiés. Souvent, ces messages cachés entrent en contradiction avec ceux qui sont ouvertement présentés; ainsi, il s'agira de considérer les relations existant entre eux afin de pouvoir comprendre le sens général de la communication. Nous avons essayé, dans le cadre de cette recherche, d'évaluer les relations existant entre certains messages cachés et certains messages déclarés transmis par les médias étudiés.

1. Message déclaré: la LJD est tout à fait abominable (même si jamais sérieusement analysée, on se réfêre normalement à la LJD en termes négatifs).

Message caché: n'importe quelle alternative est meilleure que la LJD et, par conséquent (comme par enchantement), la LJC doit être valable.

2. Message déclaré: la LJC assure des droits fondamentaux aux adolescents arrêtés, de même qu'elle expose les procédures du tribunal au public afin de permettre un plus grand contrôle social sur les juges et protège les adolescents contre les abus éventuels. La loi respecte donc la dignité ainsi que l'autonomie des jeunes contrevenants.

Message caché: la nouvelle loi présentant une approche beaucoup plus civilisée, humanitaire et éclairée, elle n'est, par conséquent, pas répressive, ni n'est-elle vengeresse, discriminatoire ou même autoritaire.

3. Message déclaré: d'abord il fut clairement déclaré, en 19801981, que la LJC serait sanctionnée. Plus tard, l'emphase fut transférée sur le fait qu'elle avait déjà été promulguée.

Message caché: quoi qu'il en soit, le Bill sera promulgué: autant s'y faire maintenant. il est inutile de remettre en question sa philosophie ou ses objets fondamentaux, néanmoins, nous pouvons toujours débattre certains détails ou, en dernière instance, les aspects financiers et techniques de son application.

4. Message déclaré: quelques problèmes tels l'âge de la responsabilité criminelle, l'aide juridique, l'interprétation de certains articles de la loi, les coûts, etc. peuvent être discutés. 
Message caché: il n’existe aucun autre problème.

5. Message déclaré: la nouvelle loi est importante en ce que la délinquance des jeunes est, de toute évidence, un problème sérieux.

Premier message caché: afin de traiter correctement le problème de la délinquance, nous avons besoin d'une nouvelle ainsi que d'une meilleure alternative juridique. (Aucune explication n'étant donnée en ce qui concerne la façon dont la nouvelle loi devrait s'y prendre pour tenter de faire diminuer la délinquance, et si en fait c'est là son objectif ultime.)

Deuxième message caché: malgré le fait qu'il n'y ait pas d'opinions clairement formulées que la LJC puisse maitriser la délinquance, c'est tout de même ce que les gens devraient supposer (ils rempliront simplement les vides de leurs propres hypothèses, de leurs attentes et de leurs interprétations). Par conséquent, on crée l'attente positive de voir le Bill diminuer la participation de la jeunesse à la criminalité. Ne nous inquiétons pas du chômage parmi les jeunes, des inégalités économiques, raciales et sexuelles, de la déchéance socio-économique de plusieurs familles, des enfants maltraités; des images de violence constamment véhiculées par les médias; des menaces qui pèsent sur l'identité des adolescents garçons qui sont poussés à s'intégrer socialement aux objectifs culturels «masculins" mais qui sont souvent privés des moyens pour y parvenir; des menaces pesant sur l'amour-propre des jeunes filles exposées aux attentes sociales contradictoires offertes par les modèles traditionnels et modemes du rôle de la femme. En d'autres termes, les reportages journalistiques sur la nouvelle législation créent l'illusion que quelque chose est entrepris pour combattre la délinquance ainsi que ses causes.

6. Message déclaré: la nouvelle loi est basée sur une approche nouvelle et supérieure de la justice pour les jeunes (ce message déclaré n'est accompagné d'aucune investigation des prémisses conflictuelles de la LJC).

Message caché: c'est une approche logique et bien définie. (Et pourtant, comment pouvons-nous associer l'accent mis sur des mesures alternatives de préjugement avec l'emphase nouvelle mise sur les procès en bonne et due forme? Comment pouvons-nous ouvrir au public le tribunal si nous avons l'intention de continuer à prendre en considération le contexte familial de l'adolescent ainsi que les circonstances de l'acte? Comment peut-on prétendre encourager la participation active des adolescents et de leurs parents aux procédures du tribunal lorsque est introduit dans la loi et au tribunal lui-même un jargon juridique spécialisé? 
Comment fait-on pour insister sur la nécessité de l'égalité de tous devant la loi si les moyens économiques permettant de retenir les services d'un brillant avocat deviennent de la plus haute importance? Comment pouvons-nous avancer qu'on pourrait s'attendre à une plus grande uniformité dans les sentences en copiant fídèlement le modèle du tribunal pour adultes quand nous connaissons les conséquences de la négociation de sentences ainsi que l'étendue des disparités dans les sentences pour adultes? De plus comment peut-on garantir l'égalité de traitement lorsqu'on est conscient que les mesures alternatives ainsi que les moyens et les facilités de traitements ne seront pas disponibles équitablement dans toutes les provinces?

7. Message déclaré: les droits juridiques ainsi que les responsabilités des jeunes contrevenants devraient être semblables à ceux et celles des contrevanants adultes.

Message caché: les droits et les responsabilités accordés aux jeunes gens dans d'autres domaines étant déjà similaires à ceux et celles de leurs ainés, par conséquent, il est normal que le système judiciaire pour les jeunes s'ajuste aux développements généraux de la société. Par ailleurs, et d'une façon réaliste, il peut être suggéré que la LJC introduit une nouvelle conception de l'adolescence: l'ancienne loi était basée sur la prémisse selon laquelle les adolescents n'ont pas atteint le niveau de maturité voulue et qu'ainsi, ils ne sont pas des membres responsables de la société: ils vivent comme des enfants, ils devraient bénéficier d'une justice pour les enfants. De nos jours, la période de l'enfance a été considérablement allongée et, à cause d'une éducation scolaire ellemême plus étendue dans le temps ou de la pénurie d'emplois non spécialisés, on s'attend à ce que les parents supportent la charge de leurs enfants pendant beaucoup plus longtemps. Les adolescents ont été aspirés par leur propre culture isolée, elle-même forgée selon des normes et des pressions commerciales, selon des moyens, des droits et des responsabilités économiques limités. Peut-on changer cet état simplement en imposant aux jeunes contrevenants de nouveaux droits et responsabilités? Si vraiment nous désirons modifier notre conception de l'adolescence, pourquoi commencer avec la justice criminelle et non pas avec la société dans son ensemble? Toutes ces questions ne furent jamais débattues par les médias étudiés.

8. Message déclaré: une proportion importante des individus interviewés supporte les principes régissant la LJC.

Premier message caché: il n'y a presque aucun groupe insatisfait de la nouvelle loi, et il règne un haut degré de consensus. Toute personne 
responsable est amenée à reconnaitre la supériorité et le très grand potentiel de la LJC.

Deuxième message caché: beaucoup d'efforts sont faits pour assurer des reportages journalistiques objectifs sur l'accueuil fait à la LJC; les journalistes interrogent les opinions de divers groupes, organisations et individus qu'ils encouragent à présenter leurs points de vue. En réalité, toutefois, comme il a été antérieurement démontré, ceux dont les opinions sont sollicitées tendent à représenter des secteurs très restreints de la société: le gouvernement, la législature et le système de la justice criminelle (environ $78 \%$ de tous les individus qui ont eu leurs opinions citées dans les reportages de presse étudiés). De plus, notons que les femmes interviewées représentèrent un infime pourcentage. Il apparaît, dès lors, que le droit de délimiter les questions entourant la LJC est accordé principalement aux représentants de la structure établie de l'autorité. On ne s'interroge par sur les intérêts qui ont été investis ainsi que sur le conditionnement structurel.

\section{CONCLUSIONS}

L'hypothèse I qui postulait que l'interprétation fonctionnaliste consensuelle de la nature des masse-médias n'était pas adéquate fut confirmée dans cette recherche. Les groupes les plus intéressés (les adolescents, leurs parents, les travailleurs sociaux) de même que les chercheurs et les défenseurs des droits humains n'ont pas eu vraiment une chance honnête d'exprimer leurs opinions. Les reportages journalistiques apparurent, dans l'ensemble, comme étant plutôt biaisés et à sens unique et dirigés vers la rhétorique officielle.

L'hypothèse 2 qui postulait que l'interprétation fonctionnaliste critique des masse-médias était très proche de la réalité, fut en partie confirmée. L'information sur la LJC fut relativement rare, les articles de première page ne constituèrent que $11 \%$ de tous les articles sélectionnés, et la majorité des informateurs représentaient des groupes d'intérêt ou des organisations puissantes. Toutefois, alors que presque $24 \%$ de tous les articles portant sur la LJD, la LJC et la délinquance des jeunes pointaient le fait que la délinquance est un phénomène de plus en plus répandu, que quelques-uns mentionnaient que les jeunes contrevenants sont de plus en plus jeunes, violents et sophistiqués, il n'y avait, entre ces messages et les décisions législatives clés concernant la LJC, aucune relation ou synchronisation évidente. Qui plus est, les aspects punitifs de la LJC qui auraient dû être présentés aux lecteurs se sont vu allouer une attention relativement restreinte (seulement 13 déclarations explicites furent trou- 
vées quant au fait que les jeunes délinquants devront désormais faire face à des conséquences plus sérieuses, et ceci, parmi un total de 67 articles mentionnant la LJC).

L'hypothèse 3 maintenait que l'analyse des reportages de presse sur la LJC démontrerait l'influence de certains groupes d'intérêt sur les médias ainsi que le besoin pour ces médias, de maintenir la crédibilité de la structure établie, mais qu'elle ne serait pas capable de vérifier la théorie marxiste de la structure sociale. La présente recherche a pu fournir de nombreuses évidences prouvant la véracité de la première partie de l'hypothèse; quant à sa deuxième partie, elle ne se réfêre pas à des données empiriques concrètes car elle relève plutôt d'un problème épistémologique complexe. Certes, il fut confirmé qu'une recherche empirique de ce genre ne peut pas fournir de bases suffisantes permettant de vérifier la pertinence de différentes visions de la structure sociale à l'intérieur de la catégorie plus large des perspectives critiques (soit pluraliste, soit marxiste).

Il serait peut-être bon de signaler, en faveur de l'interprétation marxiste, qu'en tout premier lieu, les médias ont eu tendance à mettre en évidence l'idée des droits égalitaires et de la responsabilité individuelle, deux éléments que certains marxistes considèrent comme importants dans l'hégémonie idéologique de la classe capitaliste. D'un autre côté, par contre, l'interprétation fonctionnaliste pluraliste peut, elle aussi, soutenir le fait que les médias sont intéressés à promouvoir ces idées qui contribueront à soutenir les intérêts des groupes influents importants ainsi qu'à faire appel aux membres de la société.

L'hypothèse 4 qui postulait que les reportages des médias concernant la LJC projetaient une conception fonctionnaliste consensuelle, a été confirmée par cette recherche, les reportages de presse étudiés créant l'image d'un consensus fondamental, avec des zones de discordances restreintes principalement à des questions de nature secondaire. La nouvelle législation fut présentée comme bénéfique à la société dans son ensemble, suggérant par Jà qu'aucun conflit d'intérêt n'existait entre les divers groupes touchés par la législation.

L'hypothèse 5 qui affirmait que la façon de traiter la LJC par les médias ne ressemblait pas à l'interprétation fonctionnaliste critique de la réalité sociale, fut confirmée. Aucune tentative n'a été faite pour identifier les intérêts particuliers des divers groupes impliqués (par exemple, l'expansion du chiffre d'affaires pour les avocats, l'augmentation du prestige des juges du tribunal pour adolescents, une augmentation probable des emplois et ressources pour les sociétés qui viennent en aide aux 
enfants afin de pouvoir s'occuper des 7-12 ans, des bénéfices politiques pour le Solliciteur général et le Parti libéral au pouvoir). Les médias prirent la LJC dans sa valeur nominale et la traitèrent avec respect. Aucune affirmation ne suggérait que sa fonction première était purement symbolique ou ritualiste, ou que les structures organisationnelles existantes étaient en mesure de neutraliser ou, au moins, de redéfinir les nouvelles formules juridiques. La nature des reportages sur le problème de la délinquance des jeunes semblait cependant poser quelques problèmes pour cette hypothèse. Alors que la délinquance des jeunes était discutée dans peu d'articles, la thèse qui veut qu'elle soit une fonction des inégalités et des contraintes structurelles (chômage, pauvreté relative, etc.) a été sérieusement débattue.

L'hypothèse 6 qui proposa que l'argumentation marxiste était absente des articles des médias sur la LJC fut également confirmée. Alors que plusieurs déclarations liaient la délinquance des jeunes au chômage et à d'autres causes structurelles, elles n'étaient jamais faites dans le contexte de l'évaluation de la LJC. De plus, ni les journalistes ni leurs interviewés n'explorèrent les aspects idéologiques de la construction et de la solution du problème.

En somme, il apparaît de par cette étude, que les interprétations critiques présentent la situation des masse-médias au Canada avec plus de pertinence que les interprétations consensuelles. D'un autre côté, le ton dominant des reportages des médias peut le mieux être décrit comme projetant une vision plutôt consensuelle que critique de la société.

\section{RÉFÉRENCES}

AUDLEY, Paul (1983), Canada's Cultural Industries. Broadcasting, Publishing, Records and Film, Toronto, James Lorimer and Co. Publ.

BALA, N. et H. LILLES (1983), La Loi sur les jeunes contrevenants annotée, Ottawa, ministère du Solliciteur général.

SOCIÉTÉ CANADIENNE DE CRIMINOLOGIE (1971), «Brief on Young Offenders Act, Bill C.192", Revue canadienne de criminologie, 13, 4, pp. 307-311.

COUSINEAU, D.F. et J.E. VEEVERS (1972), "Juvenile Justice: An Analysis of the Canadian Young Offenders Act", in C.L. Boydell et al. (édit.), Deviant Behaviour and Societal Reaction, Toronto, Holt, Rinehart and Winston, pp. 243-260.

FOX, Richard G. et Maureen J. SPENCER (1971-1972), "The Young Offenders Destigmatizing Juvenile Delinquency?», The Criminal Law Quarterly, 14, pp. 172-219. 
KAPLAN, Robert (1982), "Avant-propos", la Loi sur les jeunes contrevenants (points saillants), Canada, ministère des Approvisionnements et Services.

MacDONALD, John A. (1971), "A Critique of Bill C-192. The Young Offenders Act", Revue canadienne de criminologie, 3(2), pp. 166-180.

SIEGEL. Arthur (1983), Politics and the Media in Canada, Toronto, McGraw-Hill Ryerson Ltd.

ROYAL COMMISSION ON NEWSPAPERS (1981), Report prepared by the Commission, chaired by Tom Kent, Canada, Minister of Supply and Services. 